\title{
STRONGER FORMS OF A CLASS OF INEQUALITIES OF G. PÓLYA-G. SZEGÖ, AND L. V. KANTOROVICH
}

\author{
BY J. B. DIAZ ${ }^{1}$ AND F. T. METCALF ${ }^{2}$
}

Communicated by R. C. Buck, January 29, 1963

The purpose of this announcement is to show how an extremely simple idea leads immediately to inequalities for finite sums, definite integrals, etc., which are stronger than many popular inequalities now current in the literature (see the bibliography). The procedure will be illustrated in detail only in the case of finite sums. A suitable interpretation of exactly the same argument leads immediately to the corresponding results for definite integrals, etc., which will merely be stated without proof. It is planned that a more detailed presentation will appear elsewhere.

THEOREM 1. Let the real numbers $a_{k} \neq 0$ and $b_{k}(k=1,2, \cdots, n)$ satisfy

$$
m \leqq \frac{b_{k}}{a_{k}} \leqq M
$$

Then

$$
\sum_{k=1}^{n} b_{k}^{2}+m M \sum_{k=1}^{n} a_{k}^{2} \leqq(M+m) \sum_{k=1}^{n} a_{k} b_{k} .
$$

Equality holds if and only if in each of the $n$ inequalities (1), at least one of the equality signs holds, i.e., either $b_{k}=m a_{k}$ or $b_{k}=M a_{k}$ (where the equation may vary with $k$ ).

Proof. In order to establish the inequality (2) one need only note first that

$$
0 \leqq\left(\frac{b_{k}}{a_{k}}-m\right)\left(M-\frac{b_{k}}{a_{k}}\right) a_{k}^{2}
$$

follows directly from the hypothesis (1). Thus, summing from $k=1$ to $k=n$,

1 The research of this author was supported by the Air Force Office of Scientific Research-Grant AFOSR 62-454, and by the U.S. Naval Ordnance Laboratory, White Oak, Maryland.

2 The research of this author was supported by the U. S. Naval Ordnance Laboratory, White Oak, Maryland. 


$$
0 \leqq \sum_{k=1}^{n}\left(b_{k}-m a_{k}\right)\left(M a_{k}-b_{k}\right),
$$

which, upon expanding the product, gives the desired result. Clearly, equality holds in (3) (and hence, in (2)) if and only if each term of the summation is zero. The necessary and sufficient condition for equality in (2) follows readily.

Inequality (2), together with the obvious

$$
0 \leqq\left[\left(\sum_{k=1}^{n} b_{k}^{2}\right)^{1 / 2}-\left(m M \sum_{k=1}^{n} a_{k}^{2}\right)^{1 / 2}\right]^{2},
$$

yields immediately results of Cassels [5], Greub and Rheinboldt [6, p. 408, equation (5)] (letting $n \rightarrow \infty$ if needed), Kantorovich [9], Pólya and Szegö [14, problem 92], and Schweitzer [16].

There are several analogues of Theorem 1 above when the numbers $a_{k}$ and $b_{k}$ are allowed to be complex. Of these, only the following will be stated here.

THEOREM 1C. Let the complex numbers $a_{k} \neq 0$ and $b_{k}(k=1,2, \cdots, n)$ satisfy

$$
m \leqq \operatorname{Re}\left(\frac{b_{k}}{a_{k}}\right)+\operatorname{Im}\left(\frac{b_{k}}{a_{k}}\right) \leqq M
$$

and

Then

$$
m \leqq \operatorname{Re}\left(\frac{b_{k}}{a_{k}}\right)-\operatorname{Im}\left(\frac{b_{k}}{a_{k}}\right) \leqq M .
$$

$$
\begin{aligned}
\sum_{k=1}^{n}\left|b_{k}\right|^{2}+m M \sum_{k=1}^{n}\left|a_{k}\right|^{2} & \leqq(M+m) \operatorname{Re} \sum_{k=1}^{n} a_{k} b_{k} \\
& \leqq|M+m|\left|\sum_{k=1}^{n} a_{k} b_{k}\right| .
\end{aligned}
$$

Equality holds on the left of (6) if and only if: for each $k$ such that $\operatorname{Im}\left(b_{k} / a_{k}\right) \neq 0$, one equality sign holds in (4) and one equality sign (necessarily the "opposite" one) holds in (5); while for each $k$ such that $\operatorname{Im}\left(b_{k} / a_{k}\right)=0$, at least one of the equality signs holds in (4) (or, what is the same in this case, in (5)).

In order to establish (6) one needs only to show that (4) and (5) lead to

$$
0 \leqq \operatorname{Re}\left[\left(M a_{k}-b_{k}\right)\left(b_{k}-m \bar{a}_{k}\right)\right] .
$$

A "continuous" version of Theorem 1 is 
THEOREM 2. Let the real-valued functions $f(x)$ (never zero) and $g(x)$ be square integrable on the finite interval $a \leqq x \leqq b$. Suppose they satisfy

$$
m \leqq \frac{g(x)}{f(x)} \leqq M
$$

for all almost $a \leqq x \leqq b$. Then

$$
\int_{a}^{b} g^{2}(x) d x+m M \int_{a}^{b} f^{2}(x) d x \leqq(M+m) \int_{a}^{b} f(x) g(x) d x .
$$

Equality holds in (8) if and only if, for almost every $x$ in $[a, b]$, at least one of the equality signs holds in (7), where the equality sign in question may vary with $x$.

This last inequality, together with the obvious

$$
0 \leqq\left[\left(\int_{a}^{b} g^{2}(x) d x\right)^{1 / 2}-\left(m M \int_{a}^{b} f^{2}(x) d x\right)^{1 / 2}\right]^{2},
$$

yields immediately results of Pólya and Szegö [14, problem 93], Schweitzer [16], and Kürschák [10].

A "Hilbert space" version of Theorem 1 is

Theorem 3. Let $A$ and $B$ be permutable linear selfadjoint operators on a Hilbert space onto itself. Suppose that $A^{-1}$ exists and that

$$
m E \leqq B A^{-1} \leqq M E,
$$

where $E$ is the identity operator on the Hilbert space (and (9) is to be understood in the usual sense that $(m x, x) \leqq\left(B A^{-1} x, x\right) \leqq(M x, x)$ for all $x$ in the Hilbert space). Then

$$
B^{2}+m M A^{2} \leqq(m+M) A B
$$

that is to say,

$$
(B x, B x)+m M(A x, A x) \leqq(m+M)(A x, B x)
$$

for all $x$ in the Hilbert space. The equality sign holds in (10) if and only if $\left(M E-B A^{-1}\right)\left(B A^{-1}-m E\right)$ is the zero operator.

Proceeding as before, in connection with Theorems 1 and 2, and using the obvious

$$
0 \leqq\left\{(B x, B x)^{1 / 2}-[m M(A x, A x)]^{1 / 2}\right\}^{2},
$$

one obtains immediately results of Kantorovich [9], Greub and Rheinboldt [6]. 
Without entering into a detailed discussion, it is quite clear that the particular nature of the elements occurring in this class of inequalities, be they real numbers, operators, etc., is not essential. All that is needed to obtain inequalities of this general sort is a set of elements provided with binary operations of addition and multiplication, plus a subset of "non-negative" elements which have the property that the sum and the product of two non-negative elements is again non-negative.

\section{BIBLIOGRAPHY}

1. F. L. Bauer, A further generalization of the Kantorovic inequality, Numer. Math. 3 (1961), 117-119.

2. F. L. Bauer and A. S. Householder, Some inequalities involving the euclidean condition of a matrix, Numer. Math. 2 (1960), 308-311.

3. E. F. Beckenbach and Richard Bellman, Inequalities, Springer, Berlin, 1961; in particular pp. 44-45.

4. G. T. Cargo and O. Shisha, Bounds on ratios of means, J. Res. Nat. Bur. Standards 66B (1962), 169-170.

5. J. W. S. Cassels (see p. 330 and p. 340 of G. S. Watson, Serial correlation in regression analysis. I, Biometrika 42 (1955), 327-341).

6. W. Greub and W. Rheinboldt, On a generalization of an inequality of $L . V$. Kantorovich, Proc. Amer. Math. Soc. 10 (1959), 407-415.

7. Peter Henrici, Two remarks on the Kantorovich inequality, Amer. Math. Monthly 68 (1961), 904-906.

8. A. S. Householder and F. L. Bauer, On certain iterative methods for solving linear systems, Numer. Math. 2 (1960), 55-59.

9. L. V. Kantorovich, Funktsionalnii analyz $i$ prikladnaya matematika, Uspehi Mat. Nauk 3 (1948), 89-185; in particular pp. 142-144 (also translated from the Russian by C. D. Benster, Nat. Bur. Standards Report No. 1509, March 7, 1952; in particular pp. 106-109).

10. J. Kürschák, Math. Phys. Lapok 23 (1914), 378.

11. Marvin Marcus and A. H. Cayford, Further results on the Kantorovich inequality, Notices Amer. Math. Soc. 9 (1962), 300.

12. Marvin Marcus and N. A. Khan, Some generalizations of Kantorovich's inequality, Portugal. Math. 20 (1961), 33-38.

13. Morris Newman, Kantorovich's inequality, J. Res. Nat. Bur. Standards 64B (1959), 33-34.

14. G. P6lya and G. Szegö, Aufgaben und Lehrsätze aus der Analysis, Vol. 1, Springer, Berlin, 1925; in particular pp. 57, 213-214.

15. A. H. Schopf, On the Kantorovich inequality, Numer. Math. 2 (1960), 344-346.

16. P. Schweitzer, Egy egyenlötlenség az aritmetikai középértêkröl (An inequality concerning the arithmetic mean), Math. Phys. Lapok 23 (1914), 257-261.

17. W. G. Strang, On the Kantorovich inequality, Proc. Amer. Math. Soc. 11 (1960), 468.

UNIVERsity OF MARYLAND AND

U. S. Naval Ordnance Laboratory 